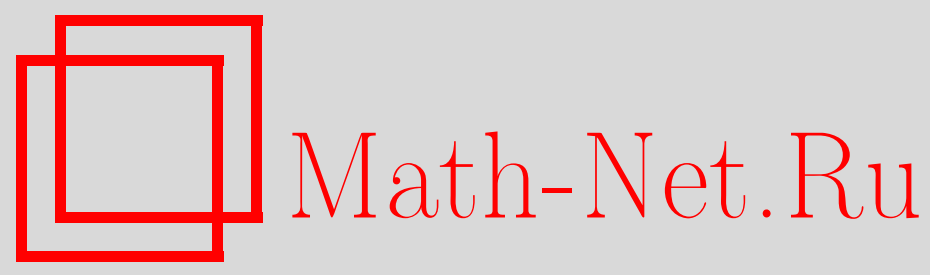

Н. Б. Керимов, Х. Р. Мамедов, О базисности Рисса корневых функций некоторых регулярных краевых задач, $M a-$ тем. заметки, 1998, том 64, выпуск 4, 558-563

DOI: https://doi.org/10.4213/mzm1430

Использование Общероссийского математического портала Math-Net.Ru подразумевает, что вы прочитали и согласны с пользовательским соглашением http://www . mathnet.ru/rus/agreement

Параметры загрузки:

IP: 3.81 .55 .215

26 апреля 2023 г., 13:45:19

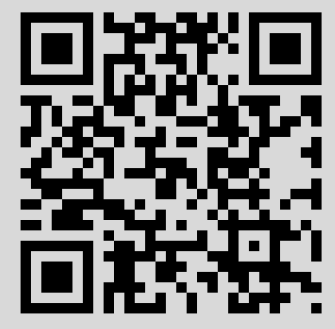




\title{
О БАЗИСНОСТИ РИССА КОРНЕВЫХ ФУНКЦИЙ НЕКОТОРЫХ РЕГУЛЯРНЫХ КРАЕВЫХ ЗАДАЧ
}

\author{
Н. Б. Керимов, Х.Р. Мамедов
}

Рассматривается дифференциальный оператор $l y=y^{\prime \prime}+q(x) y$ с периодическими (антипериодическими) краевьми условиями, являющимися не усиленно регулярньми. Предполагается, что $q(x)$ - комплекснозначная функция из класса $C^{(4)}[0,1]$ и $q(0) \neq q(1)$. Доказывается, что система корневых функций этого оператора образует базис Рисса пространства $L_{2}(0,1)$.

Библиограффия: 7 названий.

Известно [1]-[3], что система корневых функций обыкновенного дифференциального оператора произвольного порядка с усиленно регулярными краевыми условиями образует базис Рисса в $L_{2}$. В работе [2] приведен пример дифференциального оператора с регулярньми краевьми условиями (но не усиленно регулярными), корневые функции которого не образуют базис. В работах [4], [5] доказано, что система корневых функций дифференциального оператора с не усиленно регулярными краевыми условиями образует базис Рисса со скобками.

Рассмотрим дифференциальньй оператор

$$
l y=y^{\prime \prime}+q(x) y
$$

либо с периодическими краевыми условиями

$$
y(1)=y(0), \quad y^{\prime}(1)=y^{\prime}(0)
$$

либо с антипериодическими краевыми условиями

$$
y(1)=-y(0), \quad y^{\prime}(1)=-y^{\prime}(0)
$$

А. А. Шкаликов высказал гипотезу, что корневые функции задачи $(1),(2)$ или (1), (3) образуют обычньй базис Рисса, а не базис Рисса со скобками, несмотря на то, что эти задачи только регулярны, а не усиленно регулярны.

В настоящей работе мы покажем, что для этих задач свойства базисности в некоторых случаях определяются значениями потенциала в концевых точках отрезка.

Справедлива 
Tеорема. Пусть $q(x)$ - комплекснозначная функция из класса $C^{(4)}[0,1]$ u $q(1)-$ $q(0) \neq 0$. Тогда корневые функиии краевой задачи (1), (2), а также краевой задачи (1), (3) образуют базис Рисса пространства $L_{2}[0,1]$.

Лемма. Пусть $q(x)$ - комплекснозначная функиия из класса $C^{(4)}[0,1]$,

$$
q(1)-q(0) \neq 0, \quad \int_{0}^{1} q(x) d x=0
$$

Тогда справедливь следующие утверждения:

а) все собственные значения краевой задачи (1), (2), начиная с некоторого, простые и образуют две бесконечные последовательности $\lambda_{k, 1}, \lambda_{k, 2}$, $k=N, N+1, \ldots$, где $N$ - некоторое натуральное число и

$$
\begin{aligned}
& \lambda_{k, 1}=-(2 k \pi)^{2}+\frac{q(1)-q(0)}{4 k \pi}+O\left(\frac{1}{k^{2}}\right), \\
& \lambda_{k, 2}=-(2 k \pi)^{2}-\frac{q(1)-q(0)}{4 k \pi}+O\left(\frac{1}{k^{2}}\right),
\end{aligned}
$$

соответствующие собственные функиии имеют вид

$$
\begin{aligned}
& y_{k, 1}(x)=\sin 2 k \pi x-\cos 2 k \pi x+O\left(\frac{1}{k}\right), \\
& y_{k, 2}(x)=\sin 2 k \pi x+\cos 2 k \pi x+O\left(\frac{1}{k}\right)
\end{aligned}
$$

б) все собственные значения краевой задачи (1), (3), начиная с некоторого, простые и образуют две бесконечные последовательности $\lambda_{k, 1}, \lambda_{k, 2}$, $k=N, N+1, \ldots$, әде $N$ - некоторое натуральное число $и$

$$
\begin{aligned}
& \lambda_{k, 1}=-((2 k+1) \pi)^{2}-\frac{q(1)-q(0)}{2(2 k+1) \pi}+O\left(\frac{1}{k^{2}}\right), \\
& \lambda_{k, 2}=-((2 k+1) \pi)^{2}+\frac{q(1)-q(0)}{2(2 k+1) \pi}+O\left(\frac{1}{k^{2}}\right),
\end{aligned}
$$

соответствующие собственные функции имеют вид

$$
\begin{aligned}
& y_{k, 1}(x)=\sin (2 k+1) \pi x-\cos (2 k+1) \pi x+O\left(\frac{1}{k}\right) \\
& y_{k, 2}(x)=\sin (2 k+1) \pi x+\cos (2 k+1) \pi x+O\left(\frac{1}{k}\right)
\end{aligned}
$$


ДокАЗАТЕльство. Утверждения а) и б) доказываются одинаково, поэтому проведем рассуждения для а).

Рассмотрим уравнение $y^{\prime \prime}+q(x) y=\lambda y$ или

$$
y^{\prime \prime}+q(x) y+\mu^{2} y=0
$$

где $\mu=\sqrt{-\lambda}$ и $\sqrt{r e^{i \varphi}}=\sqrt{r} e^{i \varphi / 2}$ при $-\pi<\varphi \leqslant \pi$.

Известно [6, с. 74], что собственные значения краевой задачи $(1),(2)$ асимптотически расположены парами, т.е.

$$
\lambda_{k, 1}=\lambda_{k, 2}+O\left(k^{1 / 2}\right)=-(2 k \pi)^{2}\left(1+\frac{\xi_{0}}{k}+O\left(\frac{1}{k^{3 / 2}}\right)\right), \quad k=N, N+1, \ldots
$$

Из последнего равенства следует, что

$$
\begin{aligned}
& \mu_{k, 1}=\sqrt{-\lambda_{k, 1}}=2 k \pi\left(1+\frac{\xi_{0}}{2 k}+O\left(\frac{1}{k^{3 / 2}}\right)\right), \\
& \mu_{k, 2}=\sqrt{-\lambda_{k, 2}}=2 k \pi\left(1+\frac{\xi_{0}}{2 k}+O\left(\frac{1}{k^{3 / 2}}\right)\right),
\end{aligned}
$$

Следовательно, $\left|\operatorname{Im} \mu_{k, 1}\right| \leqslant c_{0},\left|\operatorname{Im} \mu_{k, 2}\right| \leqslant c_{0}$, где $c_{0}$ - некоторое положительное число.

Таким образом, при всех $k=N, N+1, \ldots$ справедливо

$$
\mu_{k, 1}, \mu_{k, 2} \in Q=\left\{\mu: \operatorname{Re} \mu \geqslant 0,|\operatorname{Im} \mu| \leqslant c_{0}\right\} .
$$

Нетрудно проверить, что $Q \in S_{0}-i c_{0} \equiv T$, где $S_{0}=\{\mu: 0 \leqslant \arg \mu \leqslant \pi / 2\}$.

Хорошо известно [6, с. 64], что в области $T$ комплексной плоскости $\mu$ уравнение $(8)$ имеет два линейно независимых решения $\varphi_{1}(x, \mu), \varphi_{2}(x, \mu)$, удовлетворяющих соотношениям

$$
\begin{gathered}
\varphi_{j}(x, \mu)=e^{\mu \omega_{j} x}\left(\sum_{m=0}^{4} \frac{u_{m}(x)}{\left(2 \omega_{j} \mu\right)^{m}}+O\left(\frac{1}{\mu^{5}}\right)\right), \\
\varphi_{j}^{\prime}(x, \mu)=\mu \omega_{j} e^{\mu \omega_{j} x}\left(u_{0}(x)+\sum_{m=1}^{4} \frac{u_{m}(x)+2 u_{m-1}^{\prime}(x)}{\left(2 \omega_{j} \mu\right)^{m}}+O\left(\frac{1}{\mu^{5}}\right)\right),
\end{gathered}
$$

где$$
\omega_{1}=-\omega_{2}=i, \quad u_{0}(x)=1, \quad u_{m}(x)=-\int_{0}^{x} l\left(u_{m-1}(\xi)\right) d \xi, \quad m=1,2,3,4 .
$$

Отсюда непосредственно следует, что

$$
\begin{aligned}
& \varphi_{j}(0, \mu)=1+O\left(\frac{1}{\mu^{5}}\right), \\
& \varphi_{j}(1, \mu)=e^{\mu \omega_{j}}\left(1+\frac{q(1)-q(0)}{\left(2 \mu \omega_{j}\right)^{2}}+\frac{1}{\left(2 \mu \omega_{j}\right)^{3}}\left(q^{\prime}(0)-q^{\prime}(1)-\int_{0}^{1} q^{2}(t) d t\right)\right. \\
& \left.+\frac{q^{\prime \prime}(1)-q^{\prime \prime}(0)+\frac{5}{2} q^{2}(1)-\frac{3}{2} q^{2}(0)-q(0) q(1)}{\left(2 \mu \omega_{j}\right)^{4}}+O\left(\frac{1}{\mu^{5}}\right)\right) \\
& \varphi_{j}^{\prime}(0, \mu)=\mu \omega_{j}\left(1-\frac{2 q(0)}{\left(2 \mu \omega_{j}\right)^{2}}+\frac{2 q^{\prime}(0)}{\left(2 \mu \omega_{j}\right)^{3}}-\frac{2 q^{\prime \prime}(0)+2 q^{2}(0)}{\left(2 \mu \omega_{j}\right)^{4}}+O\left(\frac{1}{\mu^{5}}\right)\right) \\
& \varphi_{j}^{\prime}(1, \mu)=\mu \omega_{j} e^{\mu \omega_{j}}\left(1-\frac{q(0)+q(1)}{\left(2 \mu \omega_{j}\right)^{2}}+\frac{1}{\left(2 \mu \omega_{j}\right)^{3}}\left(q^{\prime}(1)+q^{\prime}(0)-\int_{0}^{1} q^{2}(t) d t\right)\right. \\
& \left.-\frac{q^{\prime \prime}(1)+q^{\prime \prime}(0)+\frac{3}{2} q^{2}(1)+\frac{3}{2} q^{2}(0)-q(0) q(1)}{\left(2 \mu \omega_{j}\right)^{4}}+O\left(\frac{1}{\mu^{5}}\right)\right) \text {. }
\end{aligned}
$$


Подставим все эти выражения в характеристический определитель

$$
\Delta(\mu)=\left|\begin{array}{ll}
U_{1}\left(\varphi_{1}\right) & U_{1}\left(\varphi_{2}\right) \\
U_{2}\left(\varphi_{1}\right) & U_{2}\left(\varphi_{2}\right)
\end{array}\right|, \quad \text { где } \quad U_{1}(y)=y(1)-y(0), \quad U_{2}(y)=y^{\prime}(1)-y^{\prime}(0)
$$

$\mathrm{C}$ помошью элементарных преобразований находим, что при достаточно больших по абсолютной величине значениях $\mu \in T$ справедливо равенство

$$
\begin{aligned}
(i \mu)^{-1} e^{i \mu} \Delta(\mu)= & e^{2 i \mu}\left(1-\frac{2 q(0)}{(2 i \mu)^{2}}-\frac{1}{(2 i \mu)^{3}} \int_{0}^{1} q^{2}(t) d t\right. \\
& \left.-\frac{2 q^{\prime \prime}(0)-\frac{1}{2} q^{2}(1)+\frac{3}{2} q^{2}(0)+q(0) q(1)}{(2 i \mu)^{4}}+O\left(\frac{1}{\mu^{5}}\right)\right) \\
& -2 e^{i \mu}\left(1-\frac{2 q(0)}{(2 i \mu)^{2}}-\frac{2 q^{\prime \prime}(0)+2 q^{2}(0)}{(2 i \mu)^{4}}+O\left(\frac{1}{\mu^{5}}\right)\right) \\
& +\left(1-\frac{2 q(0)}{(2 i \mu)^{2}}+\frac{1}{(2 i \mu)^{3}} \int_{0}^{1} q^{2}(t) d t\right. \\
& \left.-\frac{2 q^{\prime \prime}(0)-\frac{1}{2} q^{2}(1)+\frac{3}{2} q^{2}(0)+q(0) q(1)}{(2 i \mu)^{4}}+O\left(\frac{1}{\mu^{5}}\right)\right) .
\end{aligned}
$$

Множитель при $e^{2 i \mu}$ в равенстве (9) обозначим через $b(\mu)$. Используя разложение

$$
(1-x)^{-1}=1+x+x^{2}+O\left(x^{3}\right), \quad x \rightarrow 0,
$$

нетрудно убедиться, что при больших по абсолютной величине значениях $\mu \in T$ имеет место

$$
\begin{aligned}
b^{-1}(\mu)=1 & +\frac{2 q(0)}{(2 i \mu)^{2}}+\frac{1}{(2 i \mu)^{3}} \int_{0}^{1} q^{2}(t) d t \\
& +\frac{2 q^{\prime \prime}(0)-\frac{1}{2} q^{2}(1)+\frac{11}{2} q^{2}(0)+q(0) q(1)}{(2 i \mu)^{4}}+O\left(\frac{1}{\mu^{5}}\right) .
\end{aligned}
$$

Таким образом, при больших по абсолютной величине значениях $\mu \in T$ уравнение $\Delta(\mu)=0$ равносильно уравнению

$$
(i \mu)^{-1} b^{-1}(\mu) \Delta(\mu) e^{i \mu}=0 .
$$

В силу (9), (10) уравнение (11) легко преобразовать к виду

$$
\begin{aligned}
& \left(e^{i \mu}-\left(1+\frac{1}{(2 i \mu)^{3}} \int_{0}^{1} q^{2}(t) d t-\frac{(q(1)-q(0))^{2}}{2(2 i \mu)^{4}}+O\left(\frac{1}{\mu^{5}}\right)\right)\right)^{2} \\
& =-\frac{(q(1)-q(0))^{2}}{(2 i \mu)^{4}}+O\left(\frac{1}{\mu^{5}}\right) .
\end{aligned}
$$

Поскольку $q(1)-q(0) \neq 0$, уравнение (12) распадается на два:

$$
\begin{gathered}
e^{i \mu}-1=\frac{i(q(1)-q(0))}{(2 i \mu)^{2}}+O\left(\frac{1}{\mu^{3}}\right), \\
e^{i \mu}-1=-\frac{i(q(1)-q(0))}{(2 i \mu)^{2}}+O\left(\frac{1}{\mu^{3}}\right) .
\end{gathered}
$$


Согласно теореме Руше получим асимптотические выражения для корней $\mu_{k, 1}$ и $\mu_{k, 2}$, $k=N, N+1, \ldots(N-$ некоторое натуральное число), уравнений (13) и (14) соответственно:

$$
\begin{aligned}
& \mu_{k, 1}=2 k \pi-\frac{q(1)-q(0)}{(4 k \pi)^{2}}+O\left(\frac{1}{k^{3}}\right), \\
& \mu_{k, 2}=2 k \pi+\frac{q(1)-q(0)}{(4 k \pi)^{2}}+O\left(\frac{1}{k^{3}}\right) .
\end{aligned}
$$

Отметим, что $\mu_{k, 1}$ и $\mu_{k, 2}$ - простые корни уравнений (13) и (14) соответственно. Из равенств $(15),(16)$ и соотношений $\lambda_{k, 1}=-\mu_{k, 1}^{2}, \lambda_{k, 2}=-\mu_{k, 2}^{2}$ легко получаются формулы (4) и простота этих собственных значений.

Вычислим $U_{1}\left(\varphi_{1}\left(x_{1}, \mu_{k, 1}\right)\right)$ и $U_{1}\left(\varphi_{2}\left(x_{1}, \mu_{k, 1}\right)\right)$. Поскольку

$$
e^{i \mu_{k, 1}}=1+\frac{i(q(1)-q(0))}{\left(2 i \mu_{k, 1}\right)^{2}}+O\left(\frac{1}{\mu_{k, 1}^{3}}\right),
$$

имеем

$$
\begin{aligned}
U_{1}\left(\varphi_{1}\left(x, \mu_{k, 1}\right)\right) & =\varphi_{1}\left(1, \mu_{k, 1}\right)-\varphi_{1}\left(0, \mu_{k, 1}\right) \\
& =e^{i \mu_{k, 1}}\left(1+\frac{q(1)-q(0)}{\left(2 i \mu_{k, 1}\right)^{2}}+O\left(\frac{1}{\mu_{k, 1}^{3}}\right)\right)-1-O\left(\frac{1}{\mu_{k, 1}^{5}}\right) \\
& =\frac{(1+i)(q(1)-q(0))}{\left(2 i \mu_{k, 1}\right)^{2}}+O\left(\frac{1}{\mu_{k, 1}^{3}}\right) .
\end{aligned}
$$

Аналогично получим

$$
U_{1}\left(\varphi_{2}\left(x, \mu_{k, 1}\right)\right)=\frac{(1-i)(q(1)-q(0))}{\left(2 i \mu_{k, 1}\right)^{2}}+O\left(\frac{1}{\mu_{k, 1}^{3}}\right) .
$$

Поскольку $U_{1}\left(\varphi_{j}\left(x, \mu_{k, 1}\right)\right) \neq 0, j=1,2$, и $q(1)-q(0) \neq 0$, собственную функцию $y_{k, 1}(x)$, соответствующую собственному значению $\lambda_{k, 1}$, будем искать в виде

$$
y_{k, 1}=\frac{\left(2 i \mu_{k, 1}\right)^{2}}{2 i(q(1)-q(0))}\left|\begin{array}{cc}
\varphi_{1}\left(x, \mu_{k, 1}\right) & \varphi_{2}\left(x, \mu_{k, 1}\right) \\
U_{1}\left(\varphi_{1}\left(x, \mu_{k, 1}\right)\right) & U_{1}\left(\varphi_{2}\left(x, \mu_{k, 1}\right)\right)
\end{array}\right| .
$$

Поскольку

$$
\varphi_{j}\left(x, \mu_{k, 1}\right)=e^{\mu_{k, 1} \omega_{j} x}+O\left(\frac{1}{\mu_{k, 1}}\right)
$$

(так как $\left.\left|\operatorname{Im} \mu_{k, 1}\right| \leqslant c_{0}\right)$, из формул (17)-(19) имеем

$$
y_{k, 1}=\sin \mu_{k, 1} x-\cos \mu_{k, 1} x+O\left(\frac{1}{\mu_{k, 1}}\right) .
$$

Следовательно, в силу (15) для собственной функции $y_{k, 1}(x)$ имеет место асимптотическая формула (5).

Повторяя предыдущие рассуждения для собственной функции $y_{k, 2}(x)$, соответствующей собственному значению $\lambda_{k, 2}$, получим формулу (6). Доказательство леммы завершено. 
ДоКАЗАТЕЛЬСТво тЕОРЕмЫ. Не нарушая общности, считаем, что

$$
\int_{0}^{1} q(x) d x=0
$$

Система корневых функций краевой задачи $(1),(2)$ минимальна в $L_{2}(0,1)$, поскольку обладает биортогонально сопряженной системой, состоящей из корневых функций сопряженного оператора

$$
l^{*} v=v^{\prime \prime}+\overline{q(x)} v, \quad v(0)=v(1), \quad v^{\prime}(0)=v^{\prime}(1) .
$$

Кроме того, из асимптотических формул (5), (6) следует, что система корневых функций задачи $(1),(2)$ квадратически близка к ортонормированному базису

$$
\sin 2 k \pi x-\cos 2 k \pi x, \quad k=1,2, \ldots, \quad \sin 2 k \pi x+\cos 2 k \pi x, \quad k=0,1, \ldots,
$$

пространства $L_{2}(0,1)$. Следовательно, в силу известной теоремы из $[7$, с. 440$]$ система корневых функций краевой задачи $(1),(2)$ образует базис Рисса пространства $L_{2}(0,1)$.

Аналогично доказывается утверждение теоремы для краевой задачи (1), (3). При этом используются асимптотические формулы (7).

\section{СПИСОК ЦИТИРОВАННОЙ ЛИТЕРАТУРЫ}

[1] Михайлов В.П. О базисах Рисса в $L_{2}(0,1)$ // Докл. АН СССР. 1962. Т. 144. № 5 . C. $981-984$.

[2] Кесельман Г. М. О безусловной сходимости разложений по собственным функциям некоторых дифференциальных операторов // Изв. вузов. Матем. 1964. № 2. С. 82-93.

[3] Данфорд Н., Шварц Дж. Т. Линейные операторы. Т. 3. М.: Мир, 1974.

[4] Шкаликов А. А. О базисности собственных функций обыкновенного дифференциального оператора // УМН. 1979. Т. 34. № 5. С. 235-236.

[5] Шкаликов А. А. О базисности собственных функций обыкновенного дифференциального оператора с интегральными краевьми условиями // Вестн. МГУ. Сер. 1. Матем., мех. 1982. №6. C. $12-21$.

[6] Наймарк М. А. Линейные дифференциальные операторы. М.: Наука, 1969.

[7] Качмаж С., Штейнгауз Г. Теория ортогональных рядов. М.: ИЛ, 1958.

Московский государственный университет им. М.В. Ломоносова

Поступило 29.04.96

Исправленный вариант

01.04 .98 